TRABAJO EN PROGRESO

\title{
RouteMe: Una aplicación móvil para el uso de transporte público en países en desarrollo: Caso de Tijuana
}

\author{
Adolfo De la Torre, Jesús Manuel Chaidez Avelar, \\ Lizbeth Escobedo
}

Publicado: 31 Octubre 2018

\begin{abstract}
Resumen
El uso de transporte público en ciudades con falta de infraestructura tanto vial como del propio transporte público es muy complicado. En este trabajo presentamos RouteMe, una aplicación móvil de navegación que le recomienda una ruta al usuario usando solamente las opciones de transporte público a su alcance, además. Diseñamos RouteMe con expertos y 20 usuarios de transporte público en Tijuana. Realzamos un análisis de Personas donde encontramos dos: el Veterano y el Trabajador, que caracterizan al usuario de transporte público en una ciudad como Tijuana.
\end{abstract}

Palabras clave: Notificación; Consciente del Contexto; Depresión; Recordatorio; Monitorización.

\section{Introducción}

Alrededor del mundo el transporte público terrestre es una necesidad esencial, ya que permite el desplazamiento de personas de un punto a otro. El uso eficiente del transporte público debe permitir a sus usuarios saber cómo desplazarse tomando en cuenta sus preferencias [1]. Las preferencias de los usuarios pueden variar ente menor costo, mínimo de rutas posibles, menor distancia posible, horarios, etc.

En países desarrollados como en Europa central, la infraestructura y el sistema de transporte público terrestre es muy robusto y eficiente. Lo anterior permite que se puedan desarrollar aplicaciones robustas que permitan a los usuarios saber cómo desplazarse. Existen varias aplicaciones comerciales que sugieren el transporte público que se puede utilizar en un viaje. Por ejemplo, GoogleMaps, Waze, AppleMaps, CityMapper, etc. Las sugerencias que proporcionan estas aplicaciones indican también las rutas que siguen y los horarios de llegada/partida aproximados. La conveniencia de tener la información que proporcionan estas aplicaciones es bastante útil.

Sin embargo, en países en vías de desarrollo como por ejemplo México, específicamente en la ciudad de Tijuana, la infraestructura y sistema de transporte público terrestre no es robusto. Debido a lo anterior existe un sin fin de opciones de

De la Torre, A., Escobedo, L., Chaidez, JM.

CETYS Universidad

Tijuana, México

Email: adelatorre@cetys.edu.mx, jchaidez@cetys.edu.mx,

lizbeth.escobedo@cetys.mx transporte público terrestre y que son poco convencionales (ej., existen muchas opciones de transporte público, rutas, horarios, precios, rutas incompletas-no cubiertas, etc.). Adicionalmente el transporte suele no tener un horario fijo de llegada y partida, no sigue la misma ruta siempre, y su disponibilidad cambia de día a día. Adicionalmente no todas las calles en la ciudad están etiquetadas con su nombre de calle. Todo esto ocasiona una gran confusión y molestia para los usuarios porque no es clara cuál es el transporte público que debe usar

Ante este panorama nosotros propusimos diseñar RouteMe, una aplicación móvil que permite a los usuarios desplazarse de un lugar a otro utilizando transporte público no convencional en países en desarrollo, como en el caso de la ciudad de Tijuana.

\section{Trabajo Relacionado}

Comercialmente existen varias aplicaciones populares de navegación, todas se utilizan para ayudar al usuario a desplazarse a través de una ciudad. Muchas aplicaciones incluyen horarios de transportes con horario fijo como lo son con los trenes y autobuses, otros usan información de los usuarios para dar recomendaciones sobre rutas.

GoogleMaps [2] es una aplicación de navegación altamente usada en todo el mundo que ofrece información de navegación y recomendaciones de ruta, con opciones de rutas de: carro, bicicleta, caminando y transporte público. Sin embargo, la aplicación no cubre modos de transporte público terrestre poco convencionales.

Otro ejemplo es Waze [3] que también es una aplicación de Google. Esta aplicación de navegación no solo ofrece navegación, también muchas características únicas como por ejemplo los usuarios reciben advertencias (ej., tráfico, policías, accidentes, baches, etc.) generadas por quejas recurrentes de otros usuarios

Muchas de las aplicaciones más populares son altamente usadas en países en vías de desarrollo cuando se utiliza con transporte particular, principalmente para desplazamiento, Sin embargo, todas estas aplicaciones no toman en cuenta escenarios poco convencionales como observamos en Tijuana.

\section{Diseñando RouteMe}

El proceso de diseño de RouteMe se hizo utilizando varios métodos, empezando con iterar mediante el uso de sketches el concepto de una aplicación móvil de navegación que ayuda a resolver el problema mencionado. 


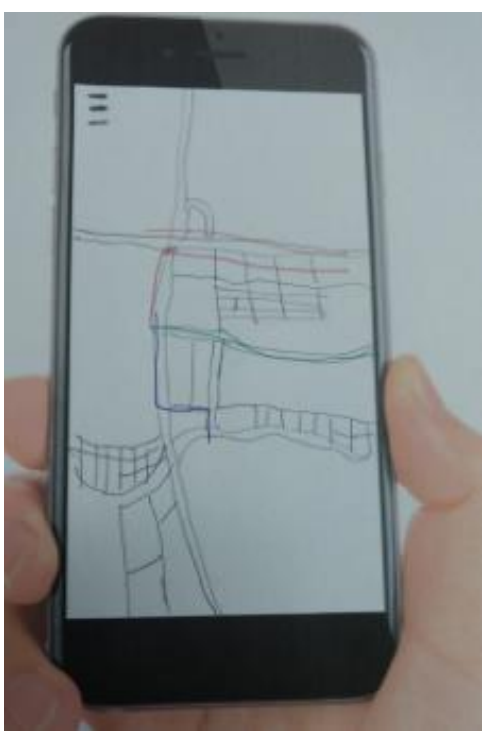

Figura 1. Sketch Hibrido que muestra la interfaz de usuario donde se despliegan 2 opciones de rutas y transporte público para desplazarse.

Después creamos las vistas que comprendían la aplicación mediante la técnica de Sketch hibrido [4] (Figura 1), para darnos una mejor idea de cómo sería la experiencia del usuario. Lo anterior también contribuyó a mejorar cada una de las vistas da la aplicación.

Con la participación de expertos en diseño, interacción humano-computadora, y desarrolladores móviles, después de varias iteraciones obtuvimos un prototipo de baja fidelidad (Figura 1). Posteriormente realizamos un prototipo de media fidelidad (Figura 2) para poder animar la experiencia del usuario, utilizando la aplicación Marvel App.

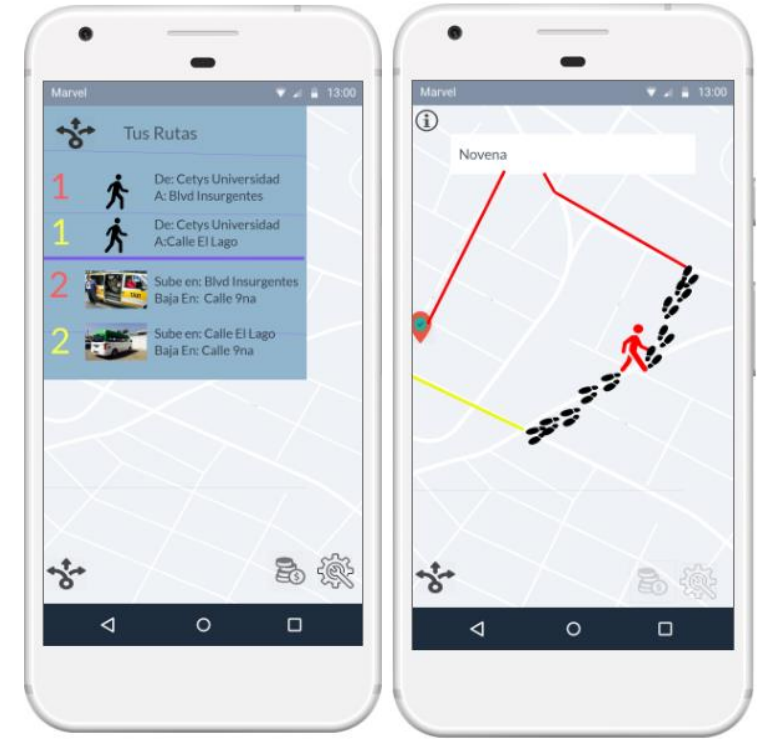

Figura 2. Prototipo de mediana fidelidad implementado en Marvel App.

RouteMe es una aplicación móvil que permite a las personas desplazarse de un lugar a otro utilizando transporte público en países en vías desarrollo. En RouteMe el usuario puede seleccionar su destino, revisar las rutas disponibles e incluso limitarlas de acuerdo con sus preferencias de costo y de lugares a evitar. Además, tiene la información de todas las formas de transporte público de la ciudad de Tijuana.

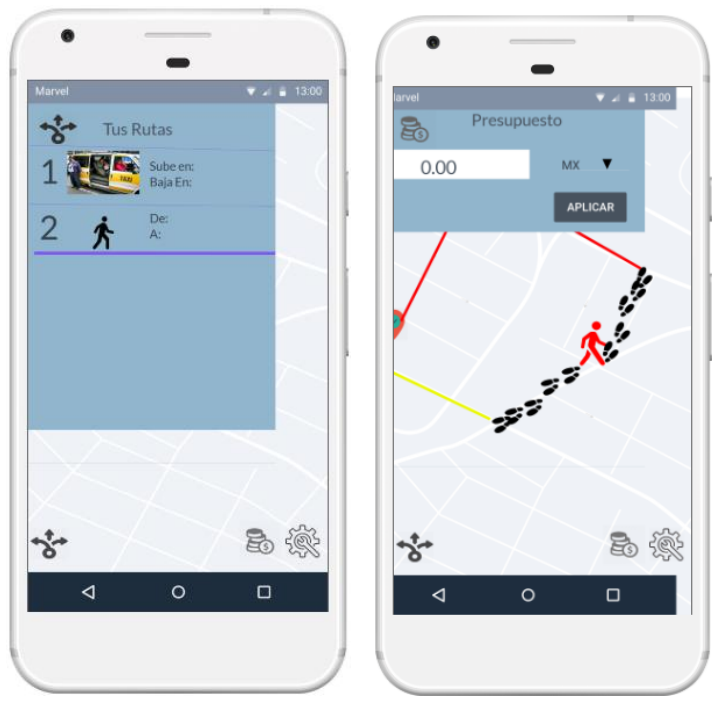

Figura 3. Vistas de opciones de ruta y preferencias de presupuesto en prototipo de Marvel App

\subsection{Análisis de Personas}

Empleamos el método de Personas [5], con el objetivo de entender a nuestros posibles usuarios y por ende mejorar nuestros diseños, entrevistamos a 20 usuarios para dicho análisis. A partir de este estudio formulamos 2 Personas:

El Veterano: Este usuario ya está familiarizado con el transporte público dado su uso, ya sea por razones personales o por profesionales, ya sabe la ruta que lo ayuda en al día y día. Pero todavía busca ahorrarse dinero cuando puede o evitar alguna zona con mala reputación.

El novato: Puede ser nuevo a la ciudad, (ejemplo un turista) puede que haya disminuido su presupuesto de transporte. Este usuario no sabe nada del transporte público local y necesita ayuda para encontrar la forma de llegar a ese lugar que todos le recomiendan.

\section{Conclusión y Futuro Trabajo}

El proceso de diseño y análisis de Personas nos hizo contar con una buena base para el diseño de RouteMe. Así mismo la técnica de Sketching y sus distintas formas (ej. tradicional, híbrido, de oficina, etc.) contribuyó también a mejorar el diseño a afinar las distintas interfaces. A futuro queremos implementar y evaluar RouteMe en un escenario real.

\section{Agradecimientos}

Especial agradecimiento a los 20 entrevistados que participaron en el estudio que nos ayudaron a hacer el análisis de Personas y mejorar el diseño de RouteMe.

\section{Referencias}

[1] Murray, A.T., Davis, R., Stimson, R.J., Ferreira L. Public Transportation Access. Transportation Research Part D: Transport and Environment, (1998), 319-328.

[2] Google Maps, https://www.google.com/maps

[3] Waze, https://www.waze.com/maps 
[4] Greenberg, S., Carpendale, S., Marquardt, N., Buxton, B. Sketching User Experiences: The Workbook, Morgan Kaufmann Publishers Inc (2011).
[5] Caddick, R., Cable, S. Communicating the user experience a practical guide for creating useful UX documentation, (2011). 